\title{
Sinistral Portal Hypertension: Clinical Features and Surgical Treatment of Chronic Splenic Vein Occlusion
}

\author{
Li Wang $^{\mathrm{a}}$ Gui-Jie Liu ${ }^{\mathrm{a}}$ Yan-Xin Chen ${ }^{\mathrm{a}}$ Huai-Ping Dong ${ }^{\mathrm{a}}$ Le-Xin Wang ${ }^{\mathrm{b}}$ \\ aDepartment of General Surgery, Liaocheng People's Hospital and Liaocheng Clinical School of

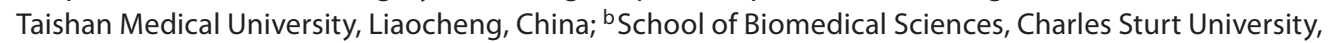 \\ Wagga Wagga, N.S.W., Australia
}

\section{Key Words}

Portal hypertension · Pancreatic diseases $\cdot$ Splenomegaly . Gastric varices, isolated $\cdot$ Splenectomy

\begin{abstract}
Objective: Sinistral, or left-sided, portal hypertension (SPH) is a rare cause of upper gastrointestinal hemorrhage. This retrospective analysis summarizes the clinical features of SPH and the results of surgical treatment. Subjects and Methods: Between 2000 and 2009, patients from our hospital diagnosed with SPH were identified. Diagnosis of SPH was based on evidence of isolated splenic vein thrombosis, splenomegaly, gastroesophageal varices and exclusion of other causes of portal hypertension. Results: Eight males and 5 females were included in the study, with a mean age of $43.5 \pm 6.4$ years (range: $31-68$ years). Liver function was normal in all patients. Causes of SPH were chronic pancreatitis $(n=7)$, pancreatic cancer $(n=3)$, pancreatic cysts $(n=2)$ and neuroendocrine tumor $(n=1)$. The main clinical manifestations were gastrointestinal hemorrhage in 7 cases (53.8\%), upper abdominal pain in 10 (76.9\%) and hypersplenism in 12 (92.3\%). All patients had splenomegaly and gastroesophageal varices. Twelve patients underwent splenectomy and 1
\end{abstract}

\section{KARGER}

Fax +4161306 1234

E-Mail karger@karger.ch

www.karger.com
() 2011 S. Karger AG, Basel

1011-7571/12/0211-0020\$38.00/0

Accessible online at:

www.karger.com/mpp received surgical removal of a pancreatic cyst. No major gastrointestinal tract rebleed occurred after a mean follow-up of 46 months ( \pm 7 months). Two patients died of pancreatic cancer and 1 of acute myocardial infarction during followup. Conclusions: SPH should be suspected in patients with upper gastrointestinal varices as well as unexplained splenomegaly with normal liver function. Surgical intervention such as splenectomy offers a good long-term outcome in symptomatic patients.

Copyright @ 2011 S. Karger AG, Basel

\section{Introduction}

Sinistral portal hypertension (SPH), also known as left-sided portal hypertension or segmental portal hypertension, is a rare cause of upper gastrointestinal hemorrhage. The main feature of SPH is gastric variceal hemorrhage due to splenic vein thrombosis or occlusion [1-4]. SPH differs from other forms of portal hypertension in that liver function is often preserved and a patent extrahepatic portal vein is present [5]. SPH is caused by pancreatic diseases, such as acute or chronic pancreatitis, pancreatic pseudocysts and pancreatic carcinomas [1-4]. 
The treatment of choice for symptomatic patients suffering recurrent variceal bleeding [6] is splenectomy; however, surgical treatment of asymptomatic patients remains controversial [5]. In this report, we describe the clinical features and outcomes of 13 patients following surgical treatment of SPH.

\section{Subjects and Methods}

Records of all patients with SPH diagnosed at Liaocheng People's Hospital between January 2000 and December 2009 were reviewed retrospectively. Diagnosis of SPH was based on evidence of isolated splenic vein thrombosis, splenomegaly and gastroesophageal varices determined by digital subtraction angiography. Splenomegaly and splenic vein thrombosis or occlusion were mainly diagnosed by Doppler ultrasonography, CT or MRI before surgical treatment [6]. Diagnostic criteria for SPH were (1) presence of pancreatic pathology, (2) clinical or laboratory evidence of portal hypertension, including total or partial occlusion of the splenic vein and splenomegaly, and (3) exclusion of other causes of portal hypertension, such as concomitant portal vein thrombosis or liver cirrhosis. Patient demographics, symptoms and signs, associated conditions, laboratory data, diagnostic evaluation, survival and new or recurrent gastrointestinal bleeding were retrieved from medical records. Follow-up was based on the last clinical evaluation in the patient's history. The study was approved by the institutional review board of Liaocheng People's Hospital. No statistical analysis was conducted since this is largely a descriptive study.

\section{Results}

Thirteen patients met the selection criteria and were included in the study. Clinical and laboratory characteristics are shown in table 1 . Seven patients had a history of serious upper gastrointestinal hemorrhage. Hypersplenism, defined as splenomegaly with thrombocytopenia, was present in 12. Chronic pancreatitis was found in 7 patients, 5 of whom also had a pancreatic pseudocyst. Of the 2 patients with pancreatic cysts in the absence of pancreatitis, one had a pseudocyst and the other had a pancreatic cystadenocarcinoma. Positive hepatic hepatitis B surface antigen was detected in 1 patient by blood test, but a liver biopsy during surgical treatment found no liver cirrhosis. Liver function tests were normal in all patients.

Apart from Doppler ultrasound and CT scans, MRI examination was performed on 6 patients, and percutaneous splenic and portal venography were conducted on 3 . These examinations revealed total occlusion of the splenic vein in 11 cases (84.6\%), and $95 \%$ stenosis of the
Table 1. Patient characteristics

\begin{tabular}{lc} 
Age, years & $43.5 \pm 6.4(31-68)$ \\
Male & $8(61.5 \%)$ \\
Etiology & \\
$\quad$ Chronic pancreatitis ${ }^{1}$ & $7(53.8 \%)$ \\
$\quad$ Pancreatic cancer & $3(23.1 \%)$ \\
$\quad$ Pancreatic cysts & $2(15.4 \%)$ \\
$\quad$ Neuroendocrine tumor & $1(7.7 \%)$ \\
Clinical manifestations before treatment & \\
Upper GI bleeding or melena & $7(53.8 \%)$ \\
Upper abdominal pain & $10(76.9 \%)$ \\
Splenomegaly & $13(100 \%)$ \\
Hypersplenism & $12(92.3 \%)$ \\
$\quad$ Reduction in blood WBC & $11(84.6 \%)$ \\
$\quad$ Reduction in blood platelets & $9(69.2 \%)$ \\
Anemia & $5(38.5 \%)$ \\
\hline
\end{tabular}

Age is given as a mean $\pm \mathrm{SD}$ with the range in parentheses. $\mathrm{GI}=$ Gastrointestinal; $\mathrm{WBC}=$ white blood cells .

${ }^{1}$ Five patients also had a pancreatic pseudocyst. ${ }^{2}$ Including 2 patients with upper gastrointestinal tract hemorrhage.

vein in 2 (15.4\%). Upper gastrointestinal digital subtraction angiography was performed on all patients. Gastroscopy was conducted on 9 . These measures showed gastroesophageal varices in all patients $(100 \%)$, including isolated gastric varices in 8 (61.5\%).

All patients underwent surgical intervention. The surgical approaches are summarized in table 2. Splenectomy was performed on 12 patients. One patient with no history of gastrointestinal hemorrhage underwent pancreatic cyst removal but no splenectomy. There was no perioperative mortality and no life-threatening complication. An increase in blood platelet count or thrombocytosis was observed in 7 patients $(53.9 \%$; mean postoperative platelet count on day 7:564 $\left.\pm 67 \times 10^{9} / 1\right)$ who received oral aspirin for 2 months to prevent thromboembolic events. Platelet numbers returned to a normal range within 2 months following the surgery. One patient (7.7\%) experienced thrombosis in the portal vein, but without clinical symptoms. The thrombus resolved after intravenous infusion of heparin for 5 days.

The mean duration of follow-up $( \pm$ SD) was $46 \pm$ 7 months, with a range from 7 to 86 months. None of the patients showed clinical symptoms or signs of gastrointestinal bleeding during follow-up. Five patients were anemic prior to surgery. Hemoglobin levels (10.9 \pm $0.6 \mathrm{~g} / \mathrm{dl})$ returned to the normal range $(13.4 \pm 0.4 \mathrm{~g} / \mathrm{dl})$ 6 months after the operation. White blood cell (ranging from 3,200 to 4,300) and platelet counts (ranging from 
Table 2. Surgical treatment

Technique

Splenectomy and pancreatectomy

Splenectomy, pancreatectomy and pericardial devascularization

Splenectomy and pericardial devascularization

Splenectomy and pancreatic duct-jejunum Roux-en-Y anastomosis

Splenectomy, pancreatic duct-jejunum Roux-en-Y anastomosis and pericardial devascularization Resection of pancreatic cyst
Number of patients

$3(20.1 \%)$

$1(7.7 \%)$

$1(7.7 \%)$

$5(38.5 \%)$

$2(15.4 \%)$

$1(7.7 \%)$
$121 \times 10^{9} / \mathrm{l}$ to $\left.139 \times 10^{9} / \mathrm{l}\right)$ were abnormal in 12 patients (including 4 patients without prior gastrointestinal bleeding) preoperatively. For each of these individuals, both white blood cell and platelet counts normalized within 6 months after surgery. Two patients with pancreatic cancer died at 16 and 23 months following surgery. Another patient died of acute myocardial infarction 40 months after surgery.

\section{Discussion}

The primary cause of SPH is thrombotic occlusion of the splenic vein [5]. Splenic vein occlusion results in back pressure, which is transmitted to the left side of the portal system through the anastomoses between the splenic vein and gastric or gastroepiploic veins. The reversal of flow in these veins results in gastric or gastroesophageal varices, which are major sources of bleeding in patients with SPH. Although both SPH and generalized portal hypertension can cause upper gastrointestinal hemorrhage, $\mathrm{SPH}$ is often associated with splenomegaly and normal liver function, as shown in the present study. Therefore, SPH should be suspected in all patients with normal liver function, splenomegaly and upper gastrointestinal bleeding.

Pancreatic disease is the most common cause of splenic thrombosis or occlusion in patients with SPH. This is largely because the splenic vein traverses the superior pancreatic surface, and is easily affected by pancreatic lesions. The most common pathologies resulting in splenic vein occlusion and SPH are chronic pancreatitis and pancreatic pseudocysts $[1,3]$. Benign or malignant pancreatic tumors account for about $18 \%$ of the SPH cases [1]. These tumors include pancreatic cancer, adenocarcinoma and islet-cell or neuroendocrine tumors [5]. Iatrogenic splenic vein injury after liver transplantation, infiltration by colonic tumor, spontaneous splenic vein throm- bosis and perirenal abscess have also been linked with $\mathrm{SPH}$ [5], but these etiological factors are rare. In the present study, more than half of the underlying pancreatic pathologies were the result of chronic pancreatitis. Pancreatic pseudocysts, including those of the 5 patients who also had chronic pancreatitis, were the second most common pathology. The third most common cause were pancreatic tumors. These results are consistent with previous reports from other investigators [1-4].

Management of SPH involves surgical correction of the underlying causes, such as pancreatic tumor or cysts, combined with splenectomy $[2,6]$. Splenectomy diminishes the arterial inflow into the left portal system and results in decompression of the gastric varices [7]. In patients with upper gastrointestinal hemorrhage, splenectomy offers clear benefits. Splenectomy in symptomatic patients is associated with correction of potentially lifethreatening bleeding, reduction of risks associated with multiple blood transfusions, prevention of hypersplenism, and possible reduction or elimination of future bleeding episodes [6]. However, there has been no consensus on the value of splenectomy in asymptomatic patients. Prophylactic splenectomy was advocated in the past, but there is the view that in patients without recurrent or life-threatening hemorrhage, initial observation and conservative management may be acceptable [5-7]. However, since portal hypertension is frequently associated with secondary hypersplenism, two common clinical manifestations of which are leukopenia and thrombocytopenia [8], patients with significant leukopenia and/or thrombocytopenia may benefit from prophylactic splenectomy.

In the present study, 7 patients (53.8\%) had a history of life-threatening gastrointestinal bleeding before surgical treatment, and 12 (92.3\%) had hypersplenism. All but 1 patient underwent splenectomy. With regard to the 7 patients suffering hemorrhage preoperatively, no recurrent gastrointestinal bleeding following surgery was 
experienced by any of them. Anemia, reduction in white blood cells and reduction in platelet counts all normalized following the surgeries. These results suggest that splenectomy is effective in preventing recurrent hemorrhage and in correcting hypersplenism-induced reduction in blood cells. Of the 5 patients with no preoperative hemorrhage, 4 had reduced white blood cell and platelet counts. Following splenectomy, the white blood cells and platelets normalized, suggesting that prophylactic splenectomy in these patients offers some clinical benefits.

\section{Conclusion}

SPH should be considered for those patients suffering upper gastrointestinal bleeding who are found to have gastric varices, signs of hypersplenism in the absence of definable hepatic injury, or cirrhosis. Chronic pancreatitis and pancreatic cysts appear to be the most common underlying pathologies for splenic vein occlusion, the key mechanism of SPH. SPH patients with upper gastrointestinal hemorrhage or with hypersplenism-induced blood cell reduction can be managed by splenectomy.

\section{References}

1 Madsen MS, Petersen TH, Sommer H: Segmental portal hypertension. Ann Surg 1986; 204:72-77.

-2 Evans GR, Yellin AE, Weaver FA, Stain SC: Sinistral (left-sided) portal hypertension. Am Surg 1990;56:758-763.

-3 Sakorafas GH, Sarr MG, Farley DR, Farnell MB: The significance of sinistral portal hypertension complicating chronic pancreatitis. Am J Surg 2000;179:129-133.
Köklü S, Yüksel O, Arhan M, Coban S, Basar O, Yolcu OF, Uçar E, Ibis M, Ertugrul I, Sahin B: Report of 24 left-sided portal hypertension cases: a single-center prospective cohort study. Dig Dis Sci 2005;50:976-982.

5 Köklü S, Coban S, Yüksel O, Arhan M: Leftsided portal hypertension. Dig Dis Sci 2007; 52:1141-1149.
Loftus JP, Nagorney DM, Illstrup D, Kunselman AR: Sinistral portal hypertension: splenectomy or expectant management. Ann Surg 1993;217:35-40.

7 Thompson RJ, Taylor MA, McKie LD, Diamond T: Sinistral portal hypertension. Ulster Med J 2006;75:175-177.

8 Pursnani KG, Sillin LF, Kaplan DS: Effect of transjugular intrahepatic portosystemic shunt on secondary hypersplenism. Am J Surg 1997;173:169-173. 